\title{
Lapurdum
}

Euskal ikerketen aldizkaria | Revue d'études basques |

Revista de estudios vascos | Basque studies review

Numéro spécial $2 \mid 2015$

Othoi çato etchera

\section{Eranskina : Euskara Île Royalen, dokumentuen argitan}

\section{Manuel Padilla-Moyano}

\section{(2) OpenEdition}

Journals

Édition électronique

URL : https://journals.openedition.org/lapurdum/2557

DOI : 10.4000/lapurdum.2557

ISSN : 1965-0655

Éditeur

IKER

Édition imprimée

Date de publication : 1 octobre 2015

Pagination : 79-83

ISBN : 978-2-9553413-1-5

ISSN : $1273-3830$

Référence électronique

Manuel Padilla-Moyano, «Eranskina : Euskara île Royalen, dokumentuen argitan», Lapurdum [Linean], Numéro spécial 2 | 2015, Sarean emana----an 25 juillet 2016, kontsultatu 02 avril 2023. URL: http:// journals.openedition.org/lapurdum/2557 ; DOI: https://doi.org/10.4000/lapurdum.2557

\section{c) (i)}

Creative Commons - Attribution-NonCommercial-NoDerivatives 4.0 International - CC BY-NC-ND 4.0 https://creativecommons.org/licenses/by-nc-nd/4.0/ 


\title{
ERANSKINA: Euskara Île Royalen, dokumentuen argitan
}

\author{
Manuel PADILLA MOYANO \\ UPV/EHU, IKER UMR5478
}

\section{Urdoz apez euskaldunaren gaineko dokumentazioa}

Frantziako Archives Nationales d'Outre-Mer (Aix-en-Provence) deitu erakundeak, besteak beste, Louisbourg hiriari lotua den dokumentazioa gordetzen du. Halako batean Urdoz apezaren gaineko bi agiri atzeman dugu, ber lotean (FR ANOM COL E382). ${ }^{1}$ Lehena Urdoz apez euskaldunaren gaineko txosten bat da, moral onaren araberako haren bizimoldea eta konduta segurtatzen duena. Bigarrenean (1714/9/24), Plazentia (Ternua) hiriko biztanleen Louisbourgerako ebakuazioaren kari, Urdoz apezari hango euskaldunekin joateko agindua ematen zaio, babesa segurtatuz. Dokumentu honek idurikatzen genuena bermatzen du: Atlantikoaz bestaldeko lapurtarrak euskaraz bizi ziren.

Quebeceko euskaldunen usantza linguistikoak oraino terra ignota baitira, Urdoz apezaren aferak galdera berriak sorrarazten dizkigu. Alde batetik, Lapurdin frantsesa aski sartua zen XVIII. mendeko, halako moduan non, funtsean, maila apaleko jendeak osatzen baitzuen euskaldun elebakarren multzoa (Oyharçabal 2001a; 2001b). Bestetik, orain bermatzen dugu Kanadako kostalde atlantikoan bizi ziren lapurtar gehienek euskara erabili ez ezik, anitzek elebakar ere jarraitzen zutela han. Baina zenbat egoiten ziren elebakar? Noraino Plazentiako euskaldunen hizkuntza ohiturak Quebecera heda litezke? Eta nola izanen zen berrogei urte berantago, hots, Le Dauphin itsasontziaren garaian?

X. Lamikizek Louisbourg-eko hizkuntza paisaia margotzen du (liburu honen 26-27. orr.). 1713an fondatutako hiria izanik, Louisbourg europarrek populatua zen. Hizkuntza erabiliena frantsesa zen, eta bigarrena euskara. Beste hizkuntzak ere erabiltzen ziren: bretoiera, alemana, gaztelania, ingelesa eta mikmakera. Horietaz gain, Frantziako Erresumako soldatapeko

1. Dokumentazio hau sarean kontsulta daiteke: <http://anom.archivesnationales.culture.gouv.fr/ ark:/61561/up424zt0yuxn> [azk. 2014/02/23]. 
mertzenarioen artean ontsalaz italiera, portugesa edo nederlandera ere mintzatuak ziratekeen. Jadanik Plazentiako euskaldunek apez euskaldun baten galdegiteak zerbait frogatzen du. Horri beste datu bat emendatzen ahal zaio: epaiketetan Louisbourgeko euskaldunek interprete bat eskatzeko eskubidea zuten, bretoiekin batean (26-27 orr.). Honek guztiak sujeritzen du egoera soziolinguistiko bat non, oro har, Louisbourgeko euskaldunek komunitate azkar bat osatzen baitzuten. Hainbat hizkuntzez inguratuta bizi arren, —edo beharbada horregatikeuskaldunak frantsesik ikasi gabe bizi zitezkeen Mundu Berrian.

Ondoan Urdoz apezaren gaineko bi piezak transkribatzen ditugu. Dokumentu originalen ortografia atxiki badugu, zenbait kasutan letra larri eta xehea egungo ohitzetara ekarri da (isle royalle $\rightarrow$ Isle Royalle); orobat unitateen arteko mugentzat (dequoy $\rightarrow$ de quoy). Bestalde, laburdura batzu garatu ditugu (lad $\rightarrow$ ladite; origl $\rightarrow$ original).

I.

$<$ lr $>$ d'Urdoz.

Philipe de Costebelle chevallier de l'Ordre Militaire de St Louiz, Gouverneur de l'Isle Royalle :

Certiffions a quy il appartiendra que le Sieur d'Urdoz, depuis plusieurs années residant dans la colonnie de Plaizance en l'Isle de Terreneuve, est un trés digne prétre, et qu'on ne peut rien luy imputér sans calomnie, et qu'a cét effect, tous les habitans et pecheurs du Pais Basque, et de Bearrix m'ayant demandé de luy ordonnér de passér a l'Isle Royalle pour leur administrér les sacrements, je le fais sans difficulté, observant neanmoins d'ezigér une requette des dits habitans, en vertu de ce que j'etois informé que Monseigneur de St Valiér éveque de Quebec avoit esté preveneu sy fort a son desavantage, qu'il avoit vouleu l'interdire sans d'autre perquisition $\langle 1 v\rangle$ de sa vie et meurs et des fonctions eclesiastiques ce que ledit Monseigneur de $S^{t}$ Valier, ayant fait a son arrivée a ladite Isle Royalle, quélle opposition que nous y ayons apporté il n’a peu excercér lesdites fonctions eclesiastiques et a esté contraint de se retirér dans son diocese de France; ce que nous nous sommes reservés de represantér a la cour pour la justiffication comme un fait dans lequel il y a reellement beaucoup d'animositté du costé de ses accuzateurs, en foy de quoy luy avons expedié le present certifficat de bonne vie et moeurs auquel pour sa validitté avons fait appozér le cachet de nos armes, ce contresignér par notre $<2$ r $>$ secretaire au port de Louisbourg dans l'Isle Royalle le $13^{\text {me }}$ octobre 1714 . Signe Costebelle plus bas par Monsieur le Gouverneur Geniér.

Collationné sur son original sans augmentér ny diminuér aucune choze, par moy Notaire Rojal a St Jean de Luz. Soussigné

$$
\text { De Valcarcel Not }{ }^{\text {aire }} \text { Royal }
$$

II.

$<3 r>$ Nous Gouverneur pour le Roy, en l'Isle Royal, et chevallier de l'Ordre Militaire de $S^{\mathrm{t}}$ Louis : 
Capitaine des vasseaux de sa Majesté, commandant le Heros, chevalier de l'Ordre Militaire de $S^{t}$ Louis de present en ce havre pour faire l'evacuation de la colomnie de Plaizance ;

Sur la representation qui nous a esté faite par les habitans de ladite colonnie que presque tous leurs equipages étoint de la nation basque, et qu'ils n'ont pas l'uzage de la langue françoise, ce consideré pour le bien du public, et de ladite colonnie ordonnons, de tous nos pouvoirs et autorités au Sieur abbé d'Urdoz de ladite nation basque quy a resté jusqu'a ce jour avec eux de passer en ladite Isle Royalle pour y faire les mémes fonctions qu'il a faite en ce lieu; Prometons audit Sieur abbé d'Urdoz de le garentir de toutes les inquietudes et empechemens ; $<3 v>$ qui luy pourront étre faits tant par les R. P. Recolets missionnaires, et curés en ladite isle que tous autres, fait a bord du Heros, le vingt quatriéme jour de septembre 1714 ; ainsy signés de Costebelle, $\&$ le chevalier de Fanion ;

Collationné sur son original sans augmentér ny diminuér aucune choze, par moy no ${ }^{\text {re }}$ rojal a St Jean de Luz,

soussigné,

$$
\text { De Valcarcel } \quad \mathrm{N}^{\mathrm{re}} \text { Royal }
$$

\section{Donibane Lohizune eta Ziburuko martxanten eta marinelen memorioa}

Ondoko dokumentua ere Archives Nationales d'Outre-Mer direlakoetan kausitzen da, COL C11C 16/no20 [bis]/6p sinatura pean. ${ }^{2}$ Bere sei orrialdeetan mamitsua da, arras informazio interesanta emanez île Royalen ziren marinel eta tratazale lapurtarren inguruan. 1733ko maiatzaren 23ko datarekin, Baionako almirantazgoko lotinantak, ofizieren izenean, Donibane Lohizune eta Ziburuko martxanten memorio bat azaltzen dio ministroari —bidenabar, lotinanta Laborde Nogués leinu ospetsuko semea da- Marinelek Île Royaleko arrantza eta merkataritza dute arrangura. Kexu nagusia da bertako bizizaleek karioegi alokatzen dizkietela itsas legartzak. Nagusiki eskatzen dute kanadar eta akadiarrekin tratatzeko libertatea eman dakiela martxantei eta kapitainei. Halaber, Erregeren funtsen banaketaren urrian egiteak ontzien partida sobera berantatzen du, horrek dakartzan ondorio gaiztoekin. Bestalde, Zacharie izeneko apez batez pleinitzen dira, berriz ere diru kontuak direla medio. Honek bide ematen die erlisioneari lotua den beste arazo baten aipatzeko: hizkuntzarena. Udaldian bi mila euskaldun izanki, eta neguan milatik gora, hauek ez ditzakete sakramentuak har, ez delako haien hizkuntza endelgatzen duen apezik. Ondorioz, euskaldunak sakramenturik gabe hiltzen dira, beren erlisionean instruitu izan gabe. Beraz, euskaraz dakien apez edo omonier baten Île Royalera igortzea eskatzen dute. Bistan da, Urdoz apezaren kasuan bezala gertatzen da 1733an: Quebeceko euskaldunek —edo haietarik anitzek—, oro har, euskaraz bizitzen

2. Dokumentua interneten kontsulta daiteke, Archives Canada-France erakundearen gunetik: <http://anom.archivesnationales.culture.gouv.fr/ark:/61561/up424zt0yuxn> [azk. 2014/02/23]. 
jarraitzen dute.

Transkripzioaz denaz bezainbatean, aitzineko dokumentazioarentzat zehaztu irizpideen pean obratu dugu.

$<$ lr $\quad$ Les officiers de l'Amirauté a Bayonne
Monseigneur
Commerce maritime
Ce memoire placé aux [?] gnaux.
de l'Isle Royale aux colonies

Ext. avec le memoire des [?] de St Jean de Luz et de Siboure

Nous avons l'honneur de remettre à votre Grandeur le memoire des negocians de $\mathrm{S}^{\mathrm{t}}$ Jean de Lus, et de Siboure sur la peche, et le commerce qu'ils font à l'Isle Royale.

Ils souhaitteroient fort, Monseigneur, que le Roy eut la bonté de fixer le loüage de la Grave, qu'ils sont obligez de prendre aux habitans de l'isle, pretendans que ces habitans ne se contentent pas de celle qui leur a été accordée par le Roy pour la secherie de leur morüe, et qu'ils en prennent au dela de ce qui leur est necessaire, pour la loüer a des prix excessifs aux vaisseaux, qui vont y faire la peche. Ils soutiennent que suivant l'usage qui se pratiquoit en Plaisance, on ne donnait à $<1 v>$ l'habitant qui loüoit sa grave, sa cabanne et son eschaffaut que dix quintaux de morüe par chaloupe équipée de trois hommes, et cinq quintaux pour chaloupe quand il loüoit seulement la grave, c'est a dire sans cabanne et sans eschaffaut, mais que comme la peche de l'Isle Royale se fait par des goeletes équipées de six a sept hommes, ces goeletes devoient étre comptées pour deux chaloupes, de sorte qu'il conviendroit qu'on fixât pour la grave chaque chaloupe a cinq quintaux, et chaque goelete ou batteau ponté a dix quintaux.

Ils avancent que le Gouverneur de Plaisance gratifioit le vaisseau qui y arrivoit le premier, à une portion de grave, outre celle qu'il avoit droit de prendre pour sa part, en consideration des secours qu'il portoit le premier à la colonie, et de la peine qu'il prenoit de distribuer, comme amiral, les places aux autres vaisseaux et ils demandent la même gratification.

Ils disent que les capitaines des deux premiers vaisseaux arrivez ont toujours joüi dans l'Eglise des droits honorifiques, soit en Plaisance, soit dans Louisbourg jusqu'en l'année 1729 que le Reverend Pere Zacharie desservant cette eglise s'y est opposé, et ils voudroient fort étre conservez dans ce droit.

$<2$ r $>$ Ils font voir qu'un des plus grands moyens d'augmenter le commerce de l'Isle Royale, est de laisser aux marchands et aux capitaines la liberté de traitter avec les canadiens, acadiens et autres sujets du Roy, et d'empecher qu'ils n'y soient troublés par 
personne, et que les officiers et les habitans n'ayent aucune prefference sur eux dans les achats, et dans les ventes. Ils esperent qu'il sera ordonné a $\mathrm{M}^{\mathrm{r}}$ le Gouverneur de Louisbourg d'y tenir la main.

Ils representent que les fonds, que le Roy envoÿe toutes les années a Louisbourg pour le payement de la garnison, et des traveaux, ne se distribuent que vers la fin d'octobre, et au mois de novembre, quoy que le vaisseau qui les porte arrive dans le mois de septembre, ce qui leur fait un tard infini par ce qu'ils sont obligez d'attendre ce tems la pour recouvrer le payement des sommes qui leur sont deües par les officiers, les entrepreneurs et les autres habitans; si cette distribution se faisoit plutôt, ils feroient plutôt leur retour en France, les equipages éviteroient le mauvais tems et les maladies, leur morüe arriveroit saine, et celle des anglois n'auroit pas dans les ports d'Espagne la prefference qu'on luy donne.

Ils parlent des droits d'amirauté, mais il paroit $<2 v>$ qu'ils confondent les droits de S.A.S. avec ceux des officiers de ladite amirauté, que nous ne connoissons pas.

Ils reviennent au R. Pere Zacharie, et ils se plaignent d'un droit qu'il a etabli de quarante sols par homme sur tous les equipages, et de l'emprisonnement qu'on a fait de leurs capitaines pour les contraindre au payement de ce droit, ce qui nous persuade que c'est par l'ordre du Roy qu'on le leve, et en ce cas la, Monseigneur, le benefice de ce religieux ne seroit pas petit.

Ils finissent en remontrant qu'il y a dans l'isle en eté deux mille hommes de ce paisci, et en hiver plus de mille, qui ne peuvent point frequenter les sacremens, par ce qu'il n'y a point de prétre qui entende leur idiome; qu'ils mourent sans les mêmes sacremens, et qu'ils ne sont nullement instruist de leur religion; qu'il seroit necessaire d'envoyer dans l'isle un pretre qui sçaut idiome Basque pour les instruire, avec le pouvoir de leur administrer les sacremens; qu'ils esperent même trouver un bone sujet, si sa majesté a la bonté de luy donner des appointemens mediocres. Voila, Monseigneur, le précis du memoire, nous vous suplions trés humblement d'etre persuadé du trés profond $<3 r>$ respect, avec lequel nous avons l'honneur d'etre.

\section{Monseigneur}

De vôtre Grandeur les trés humbles es trés obeissants serviteurs.

Laborde Nogués.

Lieutenant general en l'amiraute pour les officiers.

A Bayonne ce 23 may 1733. 